\title{
Influence of high tissue-absorbed dose on anti-thyroid antibodies in radioiodine therapy of Graves' disease patients
}

\author{
Maria Henryka Listewnik ${ }^{A, C, D}$, Hanna Piwowarska-Bilska ${ }^{\mathrm{E}}$, Krystyna Jasiakiewicz ${ }^{\mathrm{B}}$, Bożena Birkenfeld ${ }^{\mathrm{F}}$ \\ Department of Nuclear Medicine, Pomeranian Medical University, Szczecin, Poland \\ A - research concept and design; B - collection and/or assembly of data; C - data analysis and interpretation; \\ $D$ - writing the article; $E$ - critical revision of the article; $F$ - final approval of the article
}

Address for correspondence

Maria Henryka Listewnik

E-mail: listrpam@pum.edu.pl

Funding sources

None declared

Conflict of interest

None declared

Received on February 16, 2021

Reviewed on April 7, 2021

Accepted on May 19, 2021

Published online on August 19, 2021

Cite as

Listewnik MH, Piwowarska-Bilska H, Jasiakiewicz K, Birkenfeld B. Influence of high tissue-absorbed dose on anti-thyroid antibodies in radioiodine therapy of Graves' disease patients. Adv Clin Exp Med. 2021;30(9):913-921. doi:10.17219/acem/137779

DOI

10.17219/acem/137779

\section{Copyright}

Copyright by Author(s)

This is an article distributed under the terms of the

Creative Commons Attribution 3.0 Unported (CC BY 3.0)

(https://creativecommons.org/licenses/by/3.0/)

\begin{abstract}
Background. The results of radioactive iodine (RAl) treatment for Graves' disease (GD) are related to the choice of diagnostic and dosimetry protocols, the steroid protection used, and the subsequent ${ }^{131}{ }^{13}$ dose. The effect of a high tissue-absorbed dose on the level of anti-thyroid antibodies (ATA) has been rarely considered.
\end{abstract}

Objectives. To estimate the effect of the first RAl therapy with a dose of $250 \mathrm{~Gy}$ on anti-thyreoperoxidase (anti-TPO) and anti-thyroid-stimulating hormone (TSH) receptor thyrotropin receptor antibody - TRAb levels in GD patients.

Materials and methods. The analysis encompassed 46 consecutive patients with clinical presentation of GD. We examined the serum levels of TSH, free thyroxine (FT4), anti-TPO, TRAb, thyroid volume (ThV), ${ }^{131}$ I effective half-life (EHL), introduction of steroid protection, levothyroxine dose used in thyroid replacement therapy - TRT, and effectiveness of treatment.

Results. As a result of RAl treatment, hypothyroidism was found in 35 patients (76.1\%), euthyroidism in 7 patients (15.2\%) and hyperthyroidism in 4 patients (8.7\%). After RAl, we observed ThV reduction and increased anti-TPO ( $p=0.001$ and $p=0.001$, respectively). It was found that a shorter EHL correlated with a higher baseline TRAb concentration and lower final anti-TP0 serum concentration ( $p=0.03$ and $p=0.01$, respectively). Lower final TRAb was found in patients with steroid protection ( $p=0.049$ ). Intergroup comparison of patients without steroid protection showed significantly higher final anti-TPO concentation $(p=0.02)$. Intergroup comparison of patients with TRT revealed significantly higher final anti-TPO concentration $(p=0.04)$.

Conclusions. The application of a high absorbed dose of $250 \mathrm{~Gy}$ in GD resulted in high efficacy of RAl therapy at 1-year follow-up. An increased ATA level and its relationships with EHL and ThV reduction were observed at 1-year follow-up. There is a possible relationship between steroid protection and anti-TPO concentration.

Key words: hypothyroidism, Graves' disease, radioiodine therapy, thyrotropin receptor antibody (TRAb), thyroid peroxidase antibody (TPOAb) 


\section{Background}

While numerous aspects of radioactive iodine (RAI) treatment, such as its efficacy, its relation to a high absorbed dose, and its impact on clinical outcome and the shrinkage of thyroid volume (ThV) have been described in the literature, there are still some issues to be addressed. ${ }^{1}$ In view of a significant reduction of ThV after RAI therapy, one question worth considering is the influence of the therapy on the final level of anti-thyroid antibodies (ATA). ${ }^{2,3}$ Another issue to examine is the possible relationship between the level of ATA and radioiodine turnover, specifically the tracer uptake, radioactive iodine uptake - RAIU and thyroidal ${ }^{131}$ I effective half-life (EHL). ${ }^{4}$ The connection of these factors with the use of steroid protection and the potential subsequent dose of thyroid replacement therapy (TRT) due to post-therapeutic hypothyroidism is another issue to be explored. ${ }^{5}$

We believe that knowledge about the final level of ATA in patients treated with RAI due to Graves' disease (GD) is important in their follow-up. It is especially relevant in specific groups of patients, such as those with previous ophthalmopathy, of advanced age, with a history of breast cancer, or women planning a pregnancy. ${ }^{6}$ The question is whether the final evaluation of ATA after RAI therapy in patients with GD should be routinely performed. ${ }^{7}$ This paper investigates the practical aspects of using pretherapy measurements of ATA levels and the obtained dosimetric data to adjust the applied tissue-absorbed dose in case of rapid turnover. We aim to explore these factors as potentially clinically valuable information.

There are few publications describing baseline results and detailed follow-up data after RAI therapy with dosimetry data and TRT. ${ }^{8,9}$ There is also a growing interest in achieving optimal quality of life for patients after RAI. ${ }^{10,11}$

\section{Objectives}

The aim of this study was to estimate the effect of first RAI therapy with a dose of 250 Gy on anti-thyreoperoxidase (anti-TPO) and anti-thyroid-stimulating hormone (TSH) receptor (thyrotropin receptor antibody - TRAb) levels in GD patients.

\section{Materials and methods}

We present a prospective study based on data collected in 2010. The study included 46 consecutive patients (mean age: $47.4 \pm 13$ years; range: $17-72$ years, median $(\mathrm{Me})$ value of 49.5 years with interquartile range Q1-Q3 of 38.0-58.3 years with clinical presentation of GD and an elevated serum concentration of TRAb, admitted to the Department of Nuclear Medicine at Pomeranian Medical University, Szczecin, Poland. They were individually evaluated by a nuclear medicine specialist and received first RAI therapy. The study group comprised of 38 women (82.6\%; mean age: $47.1 \pm 13.4$ years, Me: $48.5(37.25-57.5)$ years, range: 17-72 years) and 8 men (7.4\%; mean age: $48.9 \pm 11.3$ years, Me: 51 (38.5-59.5) years, range: $31-62$ years).

\section{Assessment of thyroid function}

Thyroid function tests, including TSH level (reference range: $0.4-4 \mathrm{mIU} / \mathrm{L}$ ), free thyroxine (FT4) level (reference range: $10-25 \mathrm{pmol} / \mathrm{L}$ ), anti-TPO (reference range: below $60 \mathrm{IU} / \mathrm{mL}$ ), and TRAb (reference range: below $1.5 \mathrm{IU} / \mathrm{L}$ ), were performed before and 12 months after RAI therapy. The TSH level was assessed using an immunoradiometric assay, while FT4, anti-TPO and TRAb were assessed with a radioimmunoassay (TSH 1 RIA, FT4 RIA, antiTPOn RIA, TRAb Human RIA; Thermo Fisher Scientific, Waltham, USA). The laboratory criterion for recognized GD was a TRAb serum concentration level above 1.5 IU/L.

\section{Patient data}

The radioimmunoassay was applied as a first-line therapy in $2(4.3 \%)$ patients, as a second-line therapy in 42 patients (91.4\%) and as a third-line therapy in 2 patients (4.3\%). Anti-thyroid drugs were used in pre-treatment of 44 patients $(95.7 \%)$. In 10 of them, the withdrawal period was 90-360 days. In the remaining 34 patients, the withdrawal period was $17.4 \pm 16.9$ days (Me: 13 (6-60) days). No significant difference was found for ATA levels before and after RAI treatment (Mann-Whitney test for baseline anti-TPO and TRAb: $U=152, p=0.63$ and $U=161, p=0.82$, respectively; for final anti-TPO and TRAb: $U=160, p=0.79$ and $\mathrm{U}=123, \mathrm{p}=0.71$, respectively).

Aside from GD, 5 patients (10.9\%) had a comorbid autoimmune disease, including 3 (6.5\%) with rheumatoid arthritis and lupus erythematosus and 2 (4.4\%) with type 2 diabetes.

Prior to RAI therapy, all patients underwent a standard assessment of RAIU after $4 \mathrm{~h}, 24 \mathrm{~h}$ and $48 \mathrm{~h}$, and a thyroid scan with ${ }^{131}$ I after $24 \mathrm{~h}$. Thyroid volume was estimated at baseline and final assessment using planimetry phantoms. A final thyroid scan with ${ }^{99 \mathrm{~m}}$ Tc was performed after 10-12 months.

Pretherapeutic thyroid sonography was performed for all patients. A nodular pattern was found in 20 cases (43.5\%). In 4 patients $(8.7 \%)$, a complementary fine-needle aspiration biopsy was performed in selected thyroid lesions and revealed a benign origin.

Eight patients (17.4\%) were orally administered $30 \mathrm{mg}$ prednisone to prevent the exacerbation of GD orbitopathy as a routine practice, starting 1 day prior to RAI therapy. The treatment was continued for 56 consecutive days with tapering by $5 \mathrm{mg}$ every $4-5$ days according to the in-house protocol.

The inclusion criteria for steroid therapy were as follows: previous history of ophthalmopathy, younger age; and presence of a large-volume goiter. Steroid protection 
was not applied if there were comorbidities with contraindications to prednisone.

Adjuvant anti-thyroid drug therapy during follow-up was used for 5 patients (10.9\%). Within this group, 2 patients received protective corticosteroid therapy.

The ${ }^{131}$ I dose was calculated using the Marinelli formula and administered as a single oral dose in an outpatient setting. ${ }^{12}$ The radioiodine dose used was calculated according to the following formula ${ }^{13}$ :

$$
\mathrm{A}=25 \times \mathrm{m} \times \mathrm{AD} / \mathrm{RAIU} \times \mathrm{EHL}
$$

where: A - ${ }^{131}$ I therapeutic activity [MBq]; 25 - unit conversion coefficient; $\mathrm{m}$ - volume of thyroid gland using planimetry phantoms [mL]; AD - absorbed dose of ${ }^{131} \mathrm{I}$ [Gy]; RAIU - 24-hour ${ }^{131}$ I uptake [\%], and EHL - ${ }^{131}$ I effective half-life in the thyroid gland [days].

Patients were administered a dose of $463.6 \pm 188.3 \mathrm{MBq}$ (median: 400.3 (326.7-588.3) MBq, range: 177.6-969.4MBq). According to the in-house protocol, EHL estimation was based on the difference in RAIU between $24 \mathrm{~h}$ and $48 \mathrm{~h}$. The ${ }^{131} \mathrm{I}$ EHL in the thyroid is 6 days. To explain how EHL was established, we provide the following examples: if RAIU at $48 \mathrm{~h}$ and $24 \mathrm{~h}$ was $53 \%$ and $65 \%$, respectively, the difference (53\% and $65 \%$ ) was $12 \%$. In this case, we assessed EHL to be 4.8 days $(6-1.2)$. If RAIU at $48 \mathrm{~h}$ and $24 \mathrm{~h}$ was both $45 \%$, then the difference was $0(45 \%-45 \%)$ and EHL was 6 days. For patients with a RAIU of $61 \%$ at $48 \mathrm{~h}$ and $60 \%$ at $24 \mathrm{~h}$, we assessed EHL as 6.1 days $(61 \%$ - 60\%). In practice, 6 days was used as the longest EHL.

A successful outcome of RAI therapy was defined as normal thyroid function or hypothyroidism. Patients with post-therapeutic hypothyroidism were treated with a daily dose of levothyroxine sufficient to normalize TSH serum level as soon as possible. Persistent hyperthyroidism was identified as failure of radioiodine treatment.

No serious complications in thyroid eye disease were observed apart from persistent eye tearing in 3 (6.5\%) patients, who underwent corticosteroid therapy.

The study received a waiver of consent from the Bioethical Committee of the Pomeranian Medical University in Szczecin, Poland (decision No. KB-0012/202/06/17). All procedures involving human participants were in accordance with the ethical standards of the institutional and/or national research committee, and with the 1964 Declaration of Helsinki and its later amendments or comparable ethical standards.

\section{Statistical analyses}

To evaluate the normality of the distribution of the studied variables, we used the Shapiro-Wilk test. The data are reported as mean \pm standard deviation $(M \pm S D)$, range (min-max) and, due to skewed distributions, the median value with the interquartile range (Me (Q1-Q3)).

For each categorical variable, the descriptive statistics included the frequency distribution for categorical variables.
The direction and strength of association between variables were measured with Spearman's rho. We used the Mann-Whitney U two-tailed test to compare 2 independent groups and the Kruskal-Wallis $\mathrm{H}$ two-tailed test to compare 3 groups. Dunn's test of multiple comparisons following the Kruskal-Wallis test was used for post hoc analysis.

Two related matched samples were examined with the Wilcoxon signed-rank test. For categorical variables, we applied Fisher's exact test with degrees of freedom (df). All data were analyzed using STATISTICA v. 13.3 software (StatSoft, Inc, Tulsa, USA). A p-value $<0.05$ was regarded as statistically significant.

\section{Results}

Out of 46 patients, the treatment was successful in 42 patients $(91.3 \%)$. Normal thyroid function was observed in 7 patients (15.2\%) and hypothyroidism occurred in 35 patients (76.1\%). Persistent hyperthyroidism was confirmed in 4 patients (8.7\%). In the referred group, no significant difference in the mean age between females and males was found $(\mathrm{U}=139.5, \mathrm{p}=0.72)$.

The rate of average reduction of ThV after RAI therapy was $34.9 \%$. In 7 cases (15.2\%), no reduction was observed. The age and the therapeutic doses of radioiodine were comparable between patients treated with TRT and those who did not receive TRT $(\mathrm{U}=223.5, \mathrm{p}=0.83$ and $\mathrm{U}=218$, $\mathrm{p}=0.73$, respectively).

The presence of nodules did not affect the rate of hypothyroidism after RAI ( $\mathrm{df}=1, \mathrm{p}=0.49$ ). It was noted that patients in the group with thyroid nodules were older and their final TRAb levels were higher as compared to those without nodules $(53.2 \pm 11.5$ years, Me: 55.5 (46.5-59) years compared to $42.9 \pm 12.5$ years, Me: $43(34-52)$ years, $\mathrm{U}=142.5, \mathrm{p}=0.008 ; 19.3 \pm 16.6 \mathrm{IU} / \mathrm{L}, \mathrm{Me}: 13.3(4.3-40) \mathrm{IU} / \mathrm{L}$ compared to $8.2 \pm 9.5 \mathrm{IU} / \mathrm{L}, \mathrm{Me}: 3.6(1.6-12.2) \mathrm{IU} / \mathrm{L}, \mathrm{U}=116$, $\mathrm{p}=0.03)$. In the group with thyroid nodules, the final antiTPO level was significantly higher than that at baseline (2270.7 $\pm 1130.6 \mathrm{IU} / \mathrm{mL}$, Me: 2896 (2182.3-3000.0) IU/mL compared to $1554.2 \pm 1213.2 \mathrm{IU} / \mathrm{mL}$, Me: 2741.5 (308.23000) $\mathrm{IU} / \mathrm{mL}, \mathrm{Z}=2.29, \mathrm{p}=0.02$ ).

No correlation between baseline ThV and anti-TPO (rho $=0.05, \mathrm{p}=0.74$ ) or TRAb concentration ( $\mathrm{rho}=-0.07$, $\mathrm{p}=0.63)$ was observed. At final evaluation, there was no correlation between post-therapeutic ThV and anti-TPO (rho $=0.11, \mathrm{p}=0.48$ ) or TRAb concentration (rho $=-0.19$, $\mathrm{p}=0.26)$.

After RAI therapy, an increase in anti-TPO and a decrease in ThV level were found, but there were no significant differences for TRAb. A significant reduction of ThV after therapy was confirmed (Table 1).

The moderate positive correlation between the fold change of final to baseline anti-TPO and the fold change of final to baseline TRAb were both statistically significant (Fig. 1). 
Table 1. Biochemical parameters and thyroid volume (ThV) measurements at baseline and final assessment. The data are reported as mean \pm standard deviation $(\mathrm{M} \pm \mathrm{SD})$, range (min-max) and median with interquartile range (Me (Q1-Q3))

\begin{tabular}{|l|c|c|c|c|}
\hline \multicolumn{1}{|c|}{ Parameter } & Baseline assessment & Final assessment & Z-value & -value \\
\hline TSH [mIU/L] & $0.3 \pm 0.5(0.001-2.3)$ & $6.0 \pm 14.9(0.001-86.7)$ & 5.1 & $<0.001$ \\
\hline FT4 [pmol/L] & $0.1(0.02-0.2)$ & $1.5(0.5-6.4)$ & 1.7 & 0.09 \\
\hline TRAb [IU/L] & $27.2 \pm 15.7(8.4-66.1)$ & $16.5 \pm 4.8(8-25)$ & 0.34 \\
\hline Anti-TPO [IU/mL] & $20.5(15.5-38.3)$ & $17.8(12.2-18.8)$ & 0.9 & $<0.001$ \\
\hline ThV [mL] & $9.1 \pm 9.8(1.6-40)$ & $13.2 \pm 14.1(0.2-40)$ & 2.5 & $<.5$ \\
\hline
\end{tabular}

TSH - thyroid-stimulating hormone; FT4 - free thyroxine; TRAb - thyrotropin receptor antibody; Anti-TPO - anti-thyreoperoxidase; ThV - thyroid volume.

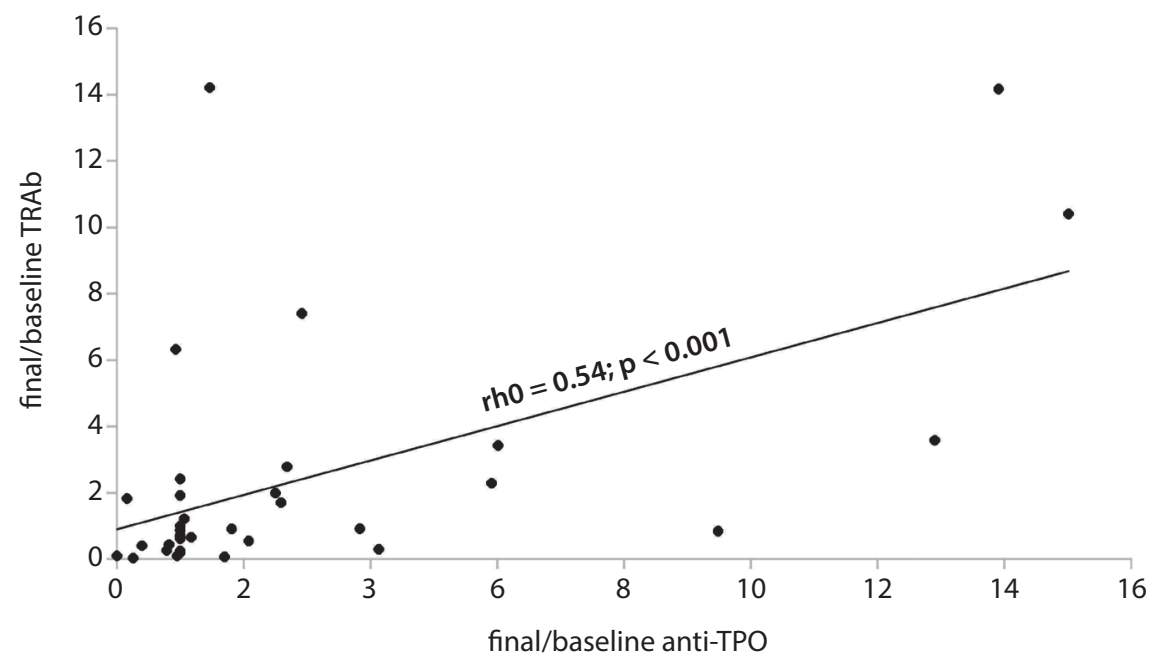

Fig. 1. Correlation between the fold change of anti-thyreoperoxidase (anti-TPO) and thyrotropin receptor antibody (TRAb) levels

\section{Dosimetry considerations}

There was a statistically significant but weak correlation between baseline TRAb levels and $4 \mathrm{~h}, 24 \mathrm{~h}$ and $48 \mathrm{~h}$ RAIU (rho $=0.38, \mathrm{p}=0.01$; rho $=0.36, \mathrm{p}=0.01$; and rho $=0.3$, $\mathrm{p}=0.04$, respectively). No relationship between baseline anti-TPO levels and RAIU was found.
We found a weak correlation between EHL and baseline TRAb, and between EHL and final anti-TPO (Fig. 2).

The total value for EHL was $5.71 \pm 0.38$ (4.8-6) days. The results of EHL assessment were categorized into 3 groups (A, B, C) based on the difference between $48 \mathrm{~h}$ and $24 \mathrm{~h}$ RAIU (range from -12 to 0 ).

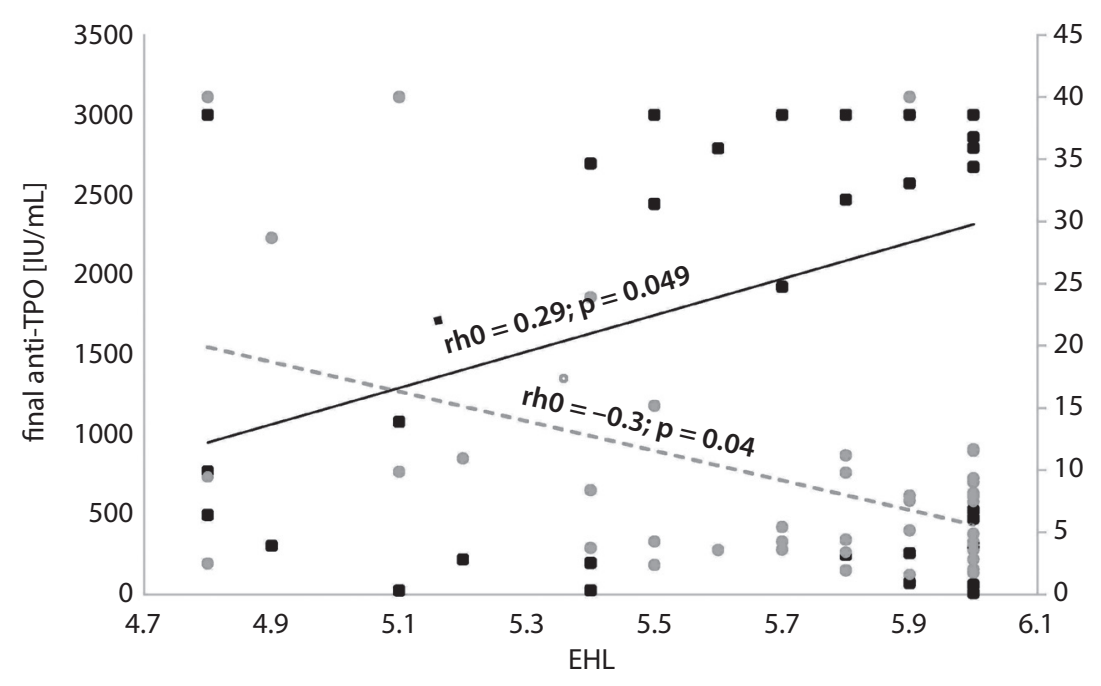

Fig. 2. Correlation between thyroidal turnover expressed as effective half-life (EHL) and final anti-thyreoperoxidase (anti-TPO) and baseline thyrotropin receptor antibody (TRAb) 


$$
11
$$


For groups A, B and C, EHL was established as $5.09 \pm 0.26$ (4.8-5.4) days, $5.75 \pm 0.15$ (5.5-5.9) days and 6 (6-6) days, respectively (Table $2 \mathrm{~A}$, Table $2 \mathrm{~B}$ ).

The categorization presented above had no impact on the final outcome $(\mathrm{df}=2, \mathrm{p}=0.25)$. There were statistically significant differences between all groups for baseline $\operatorname{TRAb}(\mathrm{H}=7.1, \mathrm{p}=0.03)$. More specifically, the significant difference was found between group $\mathrm{A}$ and $\mathrm{C}$, with higher baseline TRAb level for group A ( $p=0.03)$.

There were statistically significant differences between all groups for final anti-TPO level $(\mathrm{H}=8.7, \mathrm{p}=0.01)$. In this case, the significant difference was found between groups $\mathrm{A}$ and $\mathrm{B}$ and between groups $\mathrm{A}$ and $\mathrm{C}$, group with the lowest final anti-TPO level for group A ( $p=0.04$ and $\mathrm{p}=0.03$, respectively). The implementation of steroid protection or lack of it in all 3 EHL groups had no impact on the final assessment of (RAI) therapy (whether the patients were cured or not) $(\mathrm{df}=2, \mathrm{p}=0.61)$.

\section{Steroid protection}

No differences in hypothyroidism frequency after RAI therapy were observed between patients who did $(\mathrm{n}=8)$ or did not $(\mathrm{n}=38)$ receive steroid protection $(\mathrm{df}=1$, $\mathrm{p}=0.66$ ), as assessed with Fisher's exact test. Patients with steroid protection were younger and their baseline volume and administered therapeutic activity of ${ }^{131}$ I were higher, but their final TRAb was lower, compared to patients without glucocorticoids. Patients without glucocorticoids showed a significantly higher final anti-TPO serum concentration in the intragroup comparison (Table 3).

\section{Thyroid replacement therapy}

Out of 35 patients (76.1\%) with hypothyroidism, thyroid replacement therapy (TRT) was used in 31 (67.4\%) cases. The daily average dose of levothyroxine was $85.7 \pm 36.2 \mu \mathrm{g}$ (Me: $75(62.5-112) \mu \mathrm{g}$ ) with a range of 25-200 $\mu \mathrm{g}$.

Both groups showed significant differences in ThV before and after RAI. Final ThV was higher for patients without TRT. Comparing baseline to final data in patients with TRT, the serum concentration of anti-TPO increased. For TRAb, no differences were observed. In our study, neither baseline nor final concentrations of ATA were different between patients, irrespective of TRT (Table 4).

\section{Discussion}

The presented analysis of data on RAI therapy using a high tissue-absorbed dose of 250 Gy for 46 consecutive patients with GD, previously pretreated with an antithyroid drug indicates the need to monitor antibody levels after RAI therapy.

The study group was homogeneous in terms of both the applied absorbed dose and ATA baseline and final measurements. Although the study comprised a relatively small group of patients, many topical issues have been raised. We considered the influence of RAI on ATA with respect to ThV reduction, the usage of steroid protection, and TRT due to post-therapeutic hypothyroidism.

An absorbed dose of 250 Gy is regarded as ablative and can be increased up to 350 Gy according to the European Association of Nuclear Medicine (EANM) guidelines. ${ }^{14}$ It should be noted that some publications give the final administered ${ }^{131} \mathrm{I}$ activity value rather than the absorbed dose. ${ }^{15-20}$ Common usage of Gy (grays) to express the absorbed dose to thyroid tissue would be helpful for comparing treatment effects and analyzing the influence of particular factors. ${ }^{21}$

In our patient group, where RAI was generally a secondline therapy, $67.4 \%$ of patients had TRT at 1-year follow-up. We found no differences in baseline TRAb concentration between patients with or without subsequent TRT.

A recent long-term study by Sjölin et al. was based on a group of 1186 patients with hyperthyroidism, of whom 324 (27.3\%) had received RAI as first-line treatment, most often with an absorbed dose of $120 \mathrm{~Gy}{ }^{11}$ In the RAI therapy group, $83 \%$ of patients had hypothyroidism and $77.3 \%$ received TRT at $8 \pm 0.9$ years follow-up. Lack of dosimetry details and different choices regarding treatment make comparison impossible, but the rates of patients with TRT are slightly different, as we had $76.1 \%$ patients with hypothyroidism and $67.4 \%$ patients under TRT. However, longer follow-up could increase the rate of hypothyroidism. The rate percentage of patients with TRT (not only the total rate of hypothyroidism) is rarely taken into consideration in the literature.

The results presented in the current study are different from those of previous research, especially in terms of the rate of persistent hyperthyroidism. Aung et al. reported that $17 \%$ of patients had persistent hyperthyroidism 1 year after RAI therapy. ${ }^{16}$ Fanning et al. reported that $20.7 \%$ of patients required further treatment. ${ }^{19}$ In our group, $8.7 \%$ of patients had persistent hyperthyroidism 1 year after RAI. The achieved low failure rate proves the efficacy of RAI treatment with the use of ablative doses.

The mean patient age of 47.4 (17-72) years in our paper is almost identical to that reported in a meta-analysis by Törring et al. - a mean age of $47(35-57)$ years. ${ }^{10}$ An older age might be an independent factor for an increased level of antibodies, especially anti-TPO. ${ }^{22}$

In our group of patients, 20 (43.5\%) had nodular changes. Some authors have described the co-existence of thyroid nodules in GD and ATA in patients treated with RAI. Stoynova et al. reported a similar frequency of thyroid nodularity regardless of the TRAb level in a group previously treated with anti-thyroid drugs. ${ }^{23}$ In our group, a significant difference in TRAb levels after RAI was noted.

Mekova and Boyanov found an increased concentration of anti-TPO in $71.4 \%$ of patients without nodules among patients with newly diagnosed GD. ${ }^{24}$ In contrast to their 

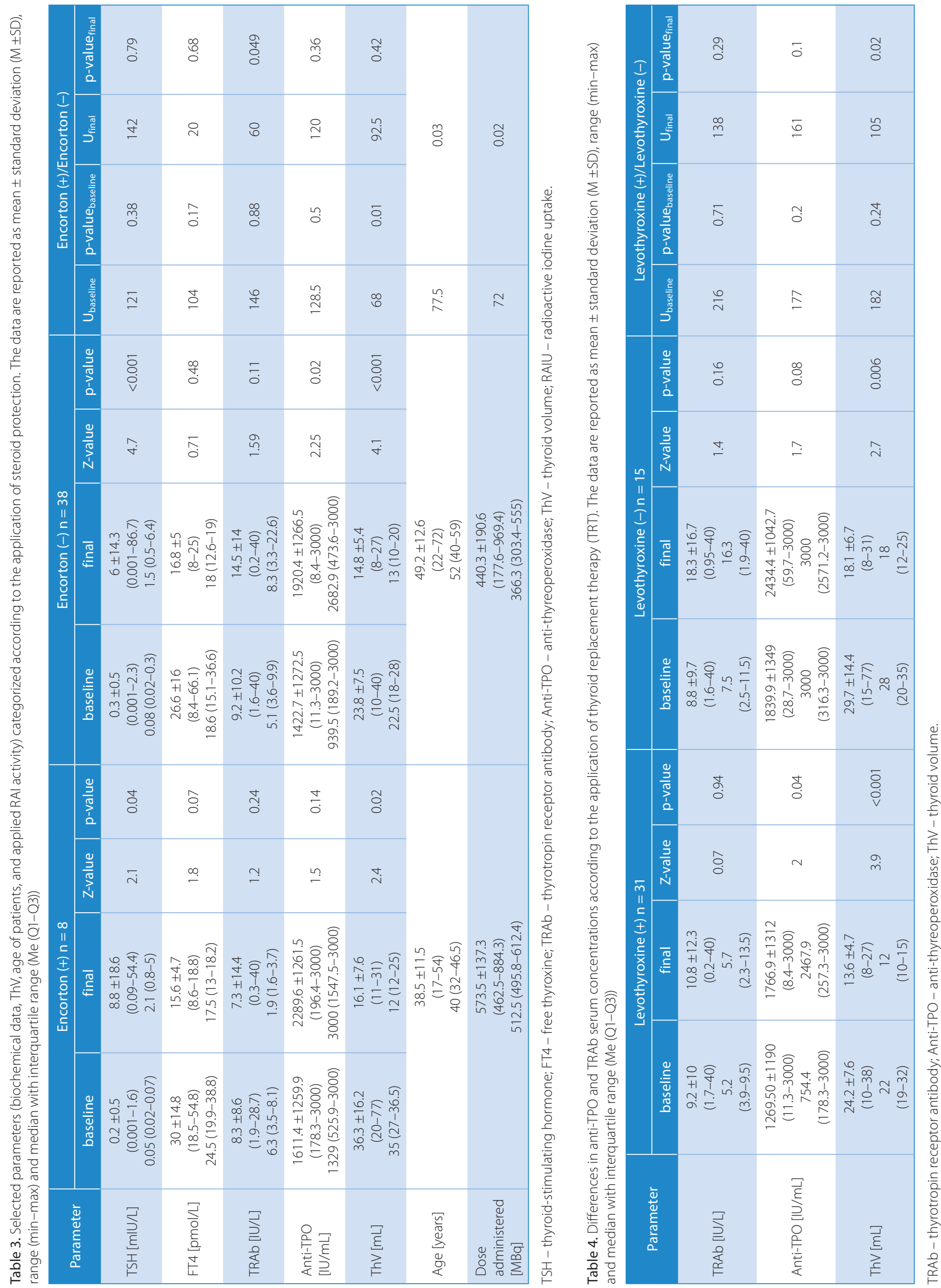
results, no differences were found in our study at baseline in nodularity and anti-TPO levels. Additionally, we examined the TRAb level in relation to the anti-TPO level. Similar to Lindgren et al., ${ }^{9}$ a positive correlation between the fold change of anti-TPO and TRAb was found. However, the absorbed dose of 120-300 Gy used by Lindgren et al. makes it difficult to compare the 2 patient groups.

Laurberg et al. also found that TRAb serum concentrations were significantly higher for RAI when compared with medical or surgical therapy, even 5 years after radioiodine therapy. ${ }^{8}$ However, Laurberg et al. applied 120 Gy to the thyroid. Similar to Laurberg et al., we observed an increased level of TRAb after 1 year. The question arises whether higher absorbed radioiodine doses, as used in our group, may cause a decrease in ATA to diminish with longer follow-up. This issue is worth investigating in the future. We also underline the importance of ATA in the case of young women planning pregnancy after RAI therapy, who should receive careful surveillance in their follow-up.

Sawicka and Sowiński reported a correlation between ThV and humoral thyroid autoimmunity after RAI therapy in GD patients. ${ }^{18}$ The majority of patients (24/36) underwent 2 RAI therapies with 12-18 months of follow-up. The authors found a significant decrease in TRAb levels, whereas our results showed no reduction in TRAb levels after RAI treatment. This difference in results may be attributed to the fact that subsequent RAI therapy may evoke a different immunological response. ${ }^{2}$

In the present paper, TRAb at baseline level correlated with $4 \mathrm{~h}, 24 \mathrm{~h}$ and $48 \mathrm{~h}$ RAIU, confirming the immunological origin of GD. This finding proves that RAIU and thyroid scanning still enable a definitive assessment of thyroid physiology. ${ }^{25}$ No such correlation was found for baseline anti-TPO. The group of patients with the highest TRAb level and the highest RAIU was characterized by the shortest EHL and the lowest anti-TPO level. In this group, the final anti-TPO level was low and stable after RAI therapy. No significant differences in the TRAb level were observed with regard to steroid protection. Additionally, volume shrinkage was noted in all groups independently of the EHL value. The mechanism of the anti-TPO reaction relies on the access of immune cells to antigens after thyrocyte destruction, whereas TRAb does not trigger such action. ${ }^{6}$ We found no explanation for this phenomenon in the literature. We hypothesize that the possible reason for the stable anti-TPO level could be the shortest EHL and the consequent shortest exposition to therapy. That is potentially why the destruction of thyroid cells was limited and, consequently, the level of anti-TPO did not increase.

Our study is not concordant with the data obtained by Aung et al. ${ }^{16}$ They showed that one of the reasons for treatment failure was higher TRAb at baseline. However, we found no impact of the baseline TRAb concentration on the success rate.

Additionally, it is known from our everyday practice that patients with the shortest EHL seem to have worse results than those with an average value of EHL. ${ }^{4}$ The study showed that, in cases of a high tissue-absorbed dose, no such effect was observed. We also found no impact of the EHL value on the success rate.

Our study also confirmed the finding reported by Jensen et al. that glucocorticoids do not have an impact on the effect of RAI therapy in GD. ${ }^{26}$ In our group of patients, those without glucocorticoid protection showed a significantly increased baseline anti-TPO level compared to the final concentration $(Z=2.25, p=0.02)$. Although the volume reaction was similar, glucocorticoids did not attenuate the immunologic reaction in anti-TPO. The reaction was similar to the one noted by Lindgren et al., who did not use glucocorticoids, but observed an increase in anti-TPO. ${ }^{9}$

With regard to TRAb, we found no significant difference in relation to a lack of steroid protection $(Z=1.59, \mathrm{p}=0.11)$. Jensen et al., who examined the influence of glucocorticoids, found no changes in the TRAb level in patients with steroid protection. ${ }^{26}$ However, it is difficult to compare the 2 datasets due to the shorter protocol time, lower dose of steroid protection and dissimilar dosimetry. Incidentally, our patients tolerated steroid protection very well and their thyroid eye disease did not deteriorate.

Differences in ATA levels after RAI therapy depending on the levothyroxine have not been widely researched in the literature. ${ }^{3,10,11}$ While the rate of hypothyroid patients after radioiodine is generally noted, authors do not usually report the fraction of patients treated with TRT. Interestingly, Sjölin et al. underlined that patients undergoing TRT were more likely to report non-recovery (as a subjective perception) than those without TRT. ${ }^{11}$ Incidentally, in our group, patients with TRT showed intragroup differences in the anti-TPO level after RAI, compared to baseline. No intergroup differences in anti-TPO and TRAb were found when the final concentrations were compared.

Damage of the thyroid parenchyma reflects the dose of RAI therapy. ${ }^{27}$ However, many factors (such as weight and age of patients, drug interactions, etc.) may determine the final dose of TRT needed. This matter requires further investigation.

The publication by Dong et al., which is based on a group of newly diagnosed patients with GD treated with RAI, is the closest to the present study. ${ }^{3}$ The patients were divided into 2 groups: those with early and non-early diagnosed post-treatment hypothyroidism. The authors came to the conclusion that serum TRAb and anti-TPO were closely related to the occurrence of early hypothyroidism and played an important role in predicting prognosis after radioiodine treatment in GD.

In nuclear medicine departments, patients are usually followed up for only 1 year. We were concerned about the observed increases in anti-TPO and TRAb levels in GD patients at discharge and decided to analyze this subject. Knowing about elevated TRAb and anti-TPO levels may help endocrinologists with patient management. Patients with increased anti-TPO and TRAb have specific 
immunological features that might occur for years. Such patients may require additional testing. Recent research suggests the protective influence of anti-TPO, for example, for extra-thyroidal pathologies in women with breast cancer. ${ }^{6}$ Despite the fact that our study was based on a small group of patients, we have identified some trends and further work on the subject is needed.

It would be interesting to design a prospective long-term study encompassing patients treated with RAI due to GD, to assess relationships between ATA and clinical and biochemical data and patient quality of life after RAI.

\section{Limitations}

Lack of information on smoking habits before and after RAI. Small number of patients. Any concentration of ATA above $40 \mathrm{IU} / \mathrm{L}$ for TRAb and above $3000 \mathrm{IU} / \mathrm{mL}$ for anti-TPO was regarded as the maximum concentration on the standard curve.

\section{Conclusions}

The application of a high absorbed dose of 250 Gy in patients with GD resulted in high RAI therapy efficacy at 1-year follow-up. An increase ATA and its relationship with EHL and ThV reduction were observed at 1-year follow-up. There is a possible relationship between steroid protection and the anti-TPO concentration.

\section{ORCID iDs}

Maria Henryka Listewnik (D) https://orcid.org/0000-0003-2801-1783 Hanna Piwowarska-Bilska (D) https://orcid.org/0000-0003-3617-7687 Krystyna Jasiakiewicz (D) https://orcid.org/0000-0002-2307-2571 Bożena Birkenfeld (D) https://orcid.org/0000-0003-1174-8199

\section{References}

1. Chiovato L, Fiore $E$, Vitti $P$, et al. Outcome of thyroid function in Graves' patients treated with radioiodine: Role of thyroid-stimulating and thyrotropin-blocking antibodies and of radioiodine-induced thyroid damage. J Clin Endocrinol Metab. 1998;83(1):40-46. doi:10. 1210/jcem.83.1.4492

2. Du W, Dong Q, Lu X, et al. lodine-131 therapy alters the immune/inflammatory responses in the thyroids of patients with Graves' disease. Exp Ther Med. 2017;13(3):1155-1159. doi:10.3892/etm.2017.4047

3. Dong Q, Liu X, Wang F, et al. Dynamic changes of TRAb and TPOab after radioiodine therapy in Graves' disease. Acta Endocrinol (Buchar). 2017;13(1):72-76. doi:10.4183/aeb.2017.72

4. Zhang R, Zhang G, Wang R, Tan J, He Y, Meng Z. Prediction of thyroidal 131 l effective half-life in patients with Graves' disease. Oncotarget. 2017;8(46):80934-80940. doi:10.18632/oncotarget.20849

5. Pyzik A, Grywalska E, Matyjaszek-Matuszek B, Roliński J. Immune disorders in Hashimoto's thyroiditis: What do we know so far? J Immunol Res. 2015;2015:979167. doi:10.1155/2015/979167

6. Fröhlich E, Wahl R. Thyroid autoimmunity: Role of anti-thyroid antibodies in thyroid and extra-thyroidal diseases. Front Immunol. 2017; 8:521. doi:10.3389/fimmu.2017.00521

7. Campi I, Vannucchi G, Covelli D, et al. Changes of serum TSH receptor antibodies (TRAB) levels in patients with Graves' disease (GD) submitted to radioiodine therapy (RAI). Eur Thyroid J. 2014;3:82. doi:10.1159/ 000365244
8. Laurberg P, Wallin G, Tallstedt L, Abraham-Nordling M, Lundell G, Tørring O. TSH-receptor autoimmunity in Graves' disease after therapy with anti-thyroid drugs, surgery, or radioiodine: A 5-year prospective randomized study. Eur J Endocrinol. 2008;158(1):69-75. doi:10. 1530/EJE-07-0450

9. Lindgren $O$, Asp P, Sundlöv A, et al. The effect of radioiodine treatment on TRAb, anti-TPO, and anti-TG in Graves' disease. Eur Thyroid J. 2019;8(2):64-69. doi:10.1159/000495504

10. Törring $O$, Watt $T$, Sjölin $G$, et al. Impaired quality of life after radioiodine therapy compared to antithyroid drugs or surgical treatment for Graves' hyperthyroidism: A long-term follow-up with the thyroidrelated patient-reported outcome questionnaire and 36-Item Short Form Health Status Survey. Thyroid. 2019;29(3):322-331. doi:10.1089/ thy.2018.0315

11. Sjölin $G$, Holmberg $M$, Törring $O$, et al. The long-term outcome of treatment for Graves' hyperthyroidism. Thyroid. 2019 29(11):1545-1557; doi:10.1089/thy.2019.0085

12. Marinelli L, Quimby E, Hine G. Dosage determination with radioactive isotopes: Practical considerations in therapy and protection. Am J Roentgenol Radium Ther. 1948;59(2):260-281. PMID:18905884

13. Szumowski P, Mojsak M, Abdelrazek S, et al. Calculation of therapeutic activity of radioiodine in Graves' disease by means of Marinelli's formula, using technetium. Endocrine. 2016;54(3):751-756. doi:10. 1007/s12020-016-1074-7

14. Stokkel MPM, Junak DH, Lassmann M, Dietlein M, Luster M. EANM procedure guidelines for therapy of benign thyroid disease. Eur J Nucl Med Mol Imaging. 2010;37(11):2218-2228. doi:10.1007/s00259-010-1536-8

15. Czarnywojtek A, Komar-Rychlicka K, Zgorzalewicz-Stachowiak M, et al. Efficacy and safety of radioiodine therapy for mild Graves ophthalmopathy depending on cigarette consumption: A 6-month followup. Pol Arch Med Wew. 2016;126(10):746-753. doi:10.20452/pamw.3505

16. Aung ET, Zammitt NN, Dover AR, Strachan MWJ, Seckl JR, Gibb FW. Predicting outcomes and complications following radioiodine therapy in Graves' thyrotoxicosis. Clin Endocrinol (Oxf). 2019;90(1):192-199. doi:10.1111/cen.13873

17. Onimode YA, Dairo DM, Ellmann A. Pattern of presentation of Graves' disease and response to radioiodine therapy in South African men. Pan Afr Med J. 2018;29:48. doi:10.11604/pamj.2018.29.48.13655

18. Sawicka N, Sowiński J. Correlation between thyroid volume and humoral thyroid autoimmunity after radioiodine therapy in Graves' disease. Endokrynol Pol. 2012;63(1):10-13. PMID:22378091

19. Fanning E, Inder WJ, Mackenzie E. Radioiodine treatment for Graves' disease: A 10-year Australian cohort study. BMC Endocr Disord. 2018; 18(1):94. doi:10.1186/s12902-018-0322-7

20. Sundaresh V, Brito JP, Wang Z, et al. Comparative effectiveness of therapies for Graves' hyperthyroidism: A systematic review and network meta-analysis. J Clin Endocrinol Metab. 2013;98(9):3671-3677. doi:10.1210/jc.2013-1954

21. Szumowski P, Abdelrazek S, Kociura Sawicka A, et al. Radioiodine therapy for Graves' disease: Retrospective analysis of efficacy factors. Endokrynol Pol. 2015;66(2):126-131. doi:10.5603/EP.2015.0019

22. Vanderpump MP. The epidemiology of thyroid disease. Br Med Bull. 2011;99:39-51. doi:10.1093/bmb/ldr030

23. Stoynova MA, Shinkov D, Kovatcheva RD, Stoynova M. Association between some ultrasound parameters and TSH-receptor antibodies. Endocrinologia. 2019;24(3):142-146.

24. Mekova R, Boyanov M. Clinical, hormonal, and ultrasound characteristics of patients with newly diagnosed Graves' disease and different thyroid antibody profiles. Acta Med Bulg. 2019;46(2):5-12. doi:10.2478/ AMB-2019-0011

25. Barbesino G, Tomer Y. Clinical review: Clinical utility of TSH receptor antibodies. J Clin Endocrinol Metab. 2013;98(6):2247-2255. doi:10.1210/ jc. 2012-4309

26. Jensen BE, Bonnema S, Hegedüs L. Glucocorticoids do not influence the effect of radioiodine therapy in Graves' disease. Eur J Endocrinol. 2005;153:15-21. doi:10.1530/eje.1.01924

27. Samadi R, Shafiei B, Azizi F, Ghasemi A. Radioactive iodine therapy and glucose tolerance. Cell J. 2017;19(2):184-193. doi:10.22074/cellj. 2016.4251 